\title{
新型二阶非线性光学生色团的合成及辅助给体对分子性能的影响研究
}

\author{
游英才 ${ }^{a}$ 唐先忠 $*, a, b$ 唐 翔 ${ }^{a, b}$ 贾鲲鹏 ${ }^{b}$ 王 洋 ${ }^{a}$ \\ 李昱树 ${ }^{a}$ 张 煜 ${ }^{a}$ \\ ( ${ }^{a}$ 电子科技大学电子薄膜与集成器件国家重点实验室 成都 610054) \\ ( ${ }^{b}$ 中国人民解放军 63916 部队 北京 100000$)$
}

\begin{abstract}
摘要 为了研究辅助给体对生色团分子热分解温度以及二阶极化率的影响, 以已报道生色团分子 2-二氧亚甲基-3-氨 基-4-(4-二乙胺基-苯乙烯基)-5,5-二甲基-2,5-二氢呋喃(DCDHF-2-V)为基础，分别在苯环二乙胺基的邻位上以羟基、丁 氧基和芐氧基作为辅助给体合成了三种新型的生色团分子: EFC-OH, EFC-OBu 和 EFC-OBe. 通过核磁共振、红外光谱 以及元素分析表征确认了其结构. 热失重分析结果表明, 三种分子的热性能良好, 其中 EFC-OBe 的热分解温度 $\left(T_{\mathrm{d}}\right)$ 最 高为 $278.6{ }^{\circ} \mathrm{C}$. 溶致变色法测定结果表明, EFC-OBe 的二阶非线性光学系数 $\left(\mu_{\mathrm{g}} \beta_{1064 \mathrm{~nm}}\right)$ 最高, 为 $47149 \times 10^{-48} \mathrm{esu}$. 将这 三种分子的 $T_{\mathrm{d}}$ 值和 $\mu_{\mathrm{g}} \beta$ 值与未加入辅助给体的 DCDHF-2-V 分子进行对比, 结果表明辅助给体的加入对分子的热稳定 性能影响不大, 但能显著提高分子的 $\mu_{g} \beta$ 值一个数量级以上.
\end{abstract}

关键词 非线性光学; 辅助给体; 热失重分析(TGA); 溶致变色法

\section{Synthesis of Novel Organic Nonlinear Optical Chromophores and the Enhancement of Electro-optic Activity of the Additional Donors}

\author{
You, Yingcai $^{a}$ \\ Tang, Xianzhong ${ }^{*, a}$ \\ Tang, Xiang ${ }^{a}$ \\ Jia, Kunpeng ${ }^{b}$ \\ Wang, Yang ${ }^{a}$ \\ $\mathrm{Li}, \mathrm{Yushu}^{a} \quad$ Zhang, $\mathrm{Yu}^{a}$ \\ ( ${ }^{a}$ State Key Laboratory of Electronic Thin Films and Integrated Devices, University of Electronic Science and \\ Technology of China, Chengdu 610054) \\ ( ${ }^{b}$ People's Liberation Army 63916 Troops, Beijing 100000)
}

\begin{abstract}
In order to study the impact of different additional donors on molecular thermal decomposition temperature $\left(T_{\mathrm{d}}\right)$ value of the chromophores as well as the second polarization $\left(\mu_{\mathrm{g}} \beta\right)$ value. Three novel materials EFC-OH, EFC-OBu and EFCOBe were synthesized by attaching additional donors to the benzene ring based on the reported 2-[\{4-(4-diethylamino)styreneyl -3-cyano-5,5-dimethyl-5H-furan-2-ylidene]malononitrile (DCDHF-2-V). The three chromophores were characterized by ${ }^{1} \mathrm{H}$ NMR, ${ }^{13} \mathrm{C}$ NHR, FT-IR and elemental analysis. The $T_{\mathrm{d}}$ value was determined by TGA testing, and the result showed that three molecules had a good thermal performance. EFC-OBe processing a $T_{\mathrm{d}}$ of $278.6{ }^{\circ} \mathrm{C}$ showed the best among these molecules. The hyperpolarizability was measured and calculated by solvatochromism method. EFC-OBe still showed the best $\mu_{\mathrm{g}} \beta$ value of $4.7149 \times 10^{-44}$ esu. Comparing to the DCDHF-2-V without additional donors on $T_{\mathrm{d}}$ value and $\mu_{\mathrm{g}} \beta$ value, additional donors had little effect on $T_{\mathrm{d}}$ value while achieved an improvement of one order of magnitude in $\mu_{\mathrm{g}} \beta$ values, which indicates that the molecules can work in high temperature and performance better in optics.

Keywords nonlinear optics; additional donor; thermogravimetic analysis (TGA); solvatochromism
\end{abstract}

目前, 有机二阶非线性光学材料的电子给体-共轭 桥-电子受体(D- $\pi$-A)电子推拉结构模型已为研究者们所 广泛认可 ${ }^{[1,2]}$, 并已合成得到很多具有良好性能的生色
团分子 ${ }^{[3 \sim 6]}$. 对于掺杂型的生色团分子而言，除了分子 本身的超极化率大小外, 分子间因聚集结晶而影响分子 取向的问题是影响材料宏观非线性光学系数的最重要

*E-mail: txzhong@uestc.edu.cn

Received September 13, 2012; revised December 12, 2012; published online December 18, 2012.

Project supported by the National Natural Science Foundation of China (No. 60771044).

国家自然科学基金(No. 60771044)资助项目. 
因素, Dalton 等 ${ }^{[7]}$ 首先发现了这一问题并通过在共轭桥 结构上引入烷基支链的方法在一定程度上解决了这一 问题. 随着对这类材料的研究不断深入, 各种优良性能 的生色团被合成出来 ${ }^{[8 \sim 11]}$, 几乎所有的一维掺杂型的有 机非线性生色团分子均是通过加入支链结构来防止分 子结晶 ${ }^{[12 \sim 14]}$.

然而, 这种将支链连接在共轭桥结构上的方法也存 在着问题. 由于这类分子的共轭桥多半是杂环结构, 而 大的支链所产生的位阻效应使得杂环的平面结构发生 扭曲, 导致电荷转移受阻, 影响了分子的非线性光学性 能 $^{[7]}$. 近来, 有研究者提出 ${ }^{[15 ~ 17]}$, 对于具有二烷氨基苯 给体的生色团, 如果将支链结构直接连接到靠给体更近 的苯环上, 而非远离给体的杂环上或许能有效地解决这 一问题. 在此基础上, 他们将具有推电子性的支链连接 在烷氨基给体的间位上, 使得支链也在整个生色团中具 有了推电子功能, 成为了 “辅助给体”, 他们的实验表 明, 辅助给体的引入, 不但不会降低分子的二阶极化率, 反而对之有所促进.

尽管如此, 目前的研究者们仅是将辅助给体引入并 研究分子的整体性能, 但是不同的辅助给体在同一种分 子中性能有何变化这一问题则鲜见报道. 基于此, 本文 在之前已有报道的具有二乙氨基苯基给体、乙烯基共轭 桥以及 TCF 受体的 DCDHF-2-V 分子 ${ }^{[18]}$ 基础上进行深入 研究, 在这种生色团分子的同一个位置(苯环上原给体 的间位)上分别引入羟基、丁氧基以及芐氧基三种结构 和性能各不相同的辅助给体, 合成了三种新型的生色团 分子, 对该生色团结构体系下, 这三种辅助给体对分子 的热性能以及非线性光学性能的影响进行了对比研究.

\section{1 结果与讨论}

\section{1 合成问题讨论}

EFC-OH, EFC-OBu, EFC-OBe ${ }^{[17]}$ 三种分子合成路线 如 Scheme 1 所示.

采用 Vilsmeier 反应合成 4- $N, N$-二乙胺基-2-羟基苯甲醛(EFO-OH). 通过 Williamson 反应合成 $4-N, N-$ 二 乙胺基-2-丁氧基-苯甲醛(EFO-OBu)和 4- $N, N$-二乙胺基2-芐氧基-苯甲醛(EFO-OBe). 酚羟基酸性较弱, 反应时 采用较弱路易斯碱 $\mathrm{K}_{2} \mathrm{CO}_{3}$ 作为催化剂. EFO-OBe 的熔点 较高, 可以在反应体系中加入冷水使其结晶为固体, 提 纯较易. EFO-OBu 的熔点较低, 在常温下为液态, 使得 反应物中剩余的 EFO-OH 溶解于 EFO-OBu 液体中, 难 以分离, 于是采用柱色谱的方法得到纯的 $\mathrm{EFO}-\mathrm{OBu}$.

采用羟醛缩合合成目标生色团分子, 三种分子在哌 啶催化下均较易生成相应的产物, 其中, EFC-OH 和 $\mathrm{EFC}-\mathrm{OBe}$ 可以直接从反应体系中结晶得到较纯的产物,
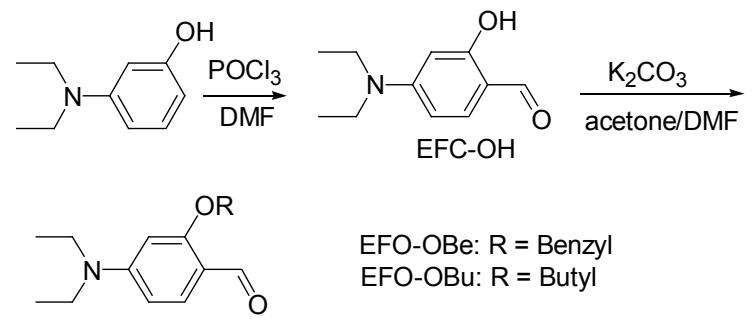

EFO-OBe: $R=$ Benzyl EFO-OBu: $R=$ Butyl
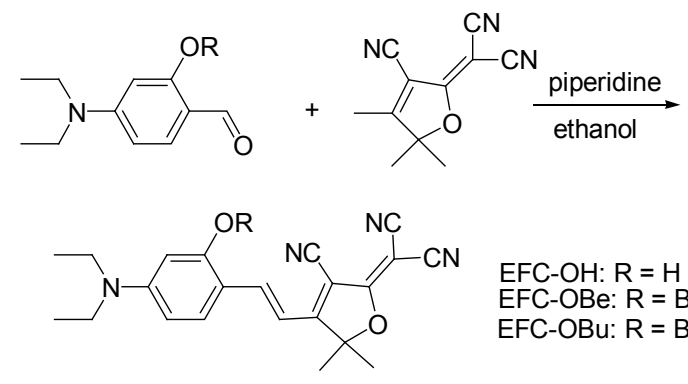

EFC-OH: $\mathrm{R}=\mathrm{H}$ EFC-OBe: $R=$ Benzyl EFC-OBu: $R=$ Butyl

\section{Scheme 1}

而 EFC-OBu 因其在乙醇中的溶解性较好, 无法直接结 晶得到产物, 需采用柱色谱提纯.

\section{2 热稳定性}

对三种生色团分子进行了热失重分析, 其 TGA 曲 线如图 1 所示.

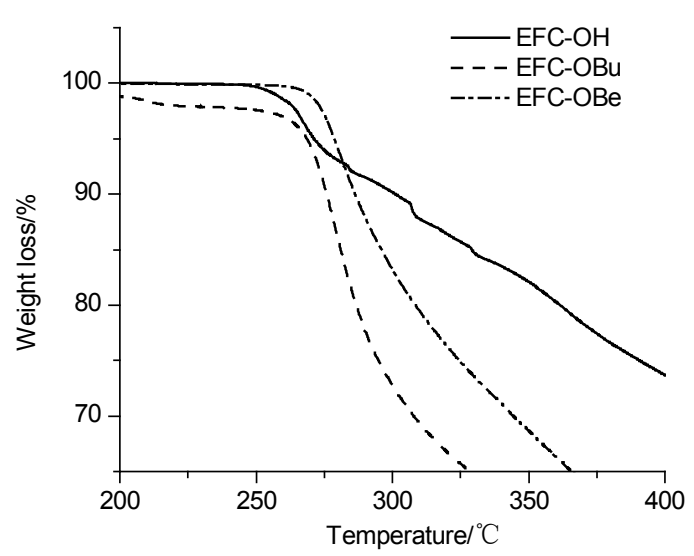

图 1 三种材料的热失重曲线图

Figure 1 TGA thermogram of the three chromophores

将实验测得三种分子的热分解温度 $T_{\mathrm{d}}$ 值 (以热失重 $5 \%$ 计)与已报道不含辅助给体的 DCDHF- $2-\mathrm{V}$ 分子 ${ }^{[18]}$ 进 行比较, 结果列于表 1 中. 对比发现, 加入辅助给体后, 三种生色团分子的 $T_{\mathrm{d}}$ 值变化不大, $\mathrm{EFC}-\mathrm{OBu}$ 最低, 比 DCDHF-2-V 分子降低了 $1.5{ }^{\circ} \mathrm{C}$; EFC-OBe 热稳定性最 好, 比未加入辅助给体时提高了 $8.6{ }^{\circ} \mathrm{C}$.

表 1 列出了目前已有报道的几种生色团分子的热分 解温度. 可以看到, 除 FTC 分子外, 一般生色团分子的 $T_{\mathrm{d}}$ 值约在 200 至 $250{ }^{\circ} \mathrm{C}$ 左右 ${ }^{[19-21]}$ 合成的三种生色团分 子具有较好的热稳定性. 
表 1 一些生色团分子的 $T_{\mathrm{d}}$ 值及 $\mu_{\mathrm{g}} \beta$ 值

Table 1 The comparison of the chromophores on $T_{\mathrm{d}}$ value and $\mu_{\mathrm{g}} \beta$ value

\begin{tabular}{lcccccccc}
\hline Chromophore & EFC-OH & EFC-OBu & EFC-OBe & DCDHF-2-V & EFNC & EFFC & FTC & A1 \\
\hline $\begin{array}{l}T_{\mathrm{d}} /{ }^{\circ} \mathrm{C} \\
\mu_{\mathrm{g}} \beta /\left(10^{-48} \mathrm{esu}\right)\end{array}$ & 271.2 & 268.5 & 278.6 & $270^{a}$ & $250^{b}$ & $250^{c}$ & $315^{d}$ & $253^{e}$ \\
$(1064 \mathrm{~nm})$ & 16910 & 24640 & 47149 & $6520^{b}$ & $59706^{b}$ & $31935^{c}$ & $17576^{d}$ & $-(1900 \mathrm{~nm})$ \\
\hline
\end{tabular}

${ }^{a}$ Data from Ref. [18]; ${ }^{b}$ Data from Ref. [23]; ${ }^{c}$ Data from Ref. [22]; ${ }^{d}$ Data from Ref. [7]; ${ }^{e}$ Data from Ref. [15].

\section{3 非线性光学性能}

$\mathrm{EFC}-\mathrm{OH}, \mathrm{EFC}-\mathrm{OBu}$ 和 $\mathrm{EFC}-\mathrm{OBe}$ 分别在乙酸乙酯, 丙酮和二甲亚砜中表现出较好的溶解性，配制精确浓度 的溶液, 分别测定三种材料在这 3 种溶剂中的紫外-可 见吸收光谱. 相关溶剂参数及实验数据见表 2 .

根据分子在不同溶剂中的紫外-可见吸收光谱以及 相关实验数据, 用溶致变色法 ${ }^{[24 ~ 26]}$ 对 $\mathrm{EFC}-\mathrm{OH}, \mathrm{EFC}-$ $\mathrm{OBu}$ 和 $\mathrm{EFC}-\mathrm{OBe}$ 分子的 $\mu_{\mathrm{g}} \beta$ 值进行了计算, 并将所得值 与已有报道的二阶非线性光学生色团进行对比, 相关数 据见表 1. 紫外-可见吸收光谱如图 2.

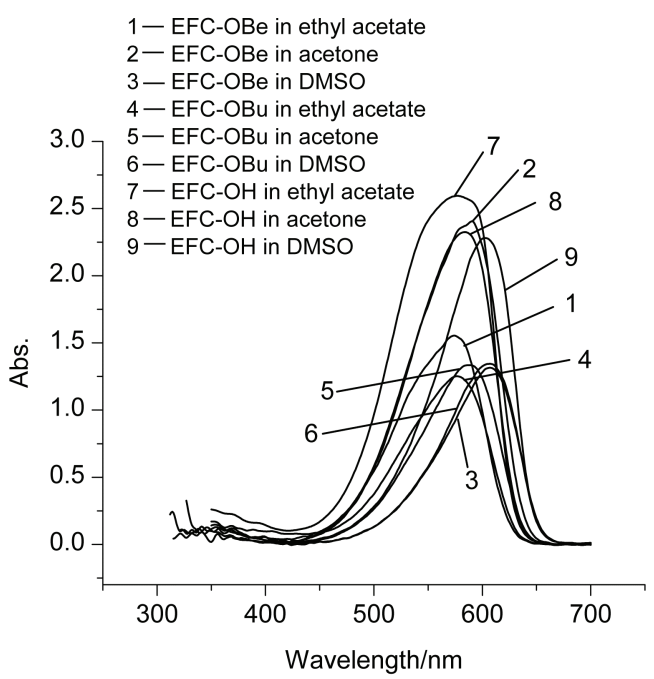

图 23 种物质在三种不同溶剂中的紫外-可见光谱图

Figure 2 UV-Vis spectra of the three chromophores in different solvents

可以看到, EFC-OH 比已经报道的 DCDHF-2-V 的 $\mu_{\mathrm{g}} \beta$ 值提高了一个数量级, 相应的, $\mathrm{EFC}-\mathrm{OBu}$ 与 $\mathrm{EFC}-$ $\mathrm{OBe}$ 均有较大幅度提高, 其中, EFC-OBe 相比 DCDHF-
$2-\mathrm{V}$ 提高最多, 达 7 倍以上, 而提高最少的 $\mathrm{EFC}-\mathrm{OH}$ 的 $\mu_{\mathrm{g}} \beta$ 值也是未加入辅助给体前的近 3 倍.

在苯环中原给体的间位上引入辅助给体后，分子的 $\mu_{g} \beta$ 值均出现了较为显著的增大, 这可能是因为在 DCDHF-2-V 分子中, 受体吸电子能力较强, 而给电子 基团的给电子能力不足以让吸电子基团达到饱和，当稍 微增加给电子能力, 就使得其非线性光学系数有了一个 较大的提升幅度 ${ }^{[22]}$. 理论上羟基给电子能力最强, 丁氧 基给电子能力最弱, 但实际上 $\mathrm{EFC}-\mathrm{OH}$ 对应 $\mu_{\mathrm{g}} \beta$ 值最小, 我们认为这可能是因为 $\mathrm{EFC}-\mathrm{OH}$ 在溶剂中存在分子间 氢键作用，分散了氧原子上的电荷密度; 也有可能是因 为溶剂化效应导致推拉电子基团的能力受到了影响，溶 剂化效应有时会使传统的 D- $\pi$-A 电荷转移体系 $\beta$ 值失 效 ${ }^{[27]}$, 使其在溶剂中表现不太良好. EFC-OBe 对应 $\mu_{g} \beta$ 值最大, $\mathrm{EFC}-\mathrm{OBu}$ 次之, 这是因为苄氧基的给电子能力 大于丁氧基.

从表 1 中 $\mu_{g} \beta$ 值的数据对比还可以看到, 单纯的苯 乙烯共轭桥生色团仅为 $10^{-45} \mathrm{esu}$ 数量级. 通过在共轭桥 中加入偶氮苯或乙烯基杂环可提高分子二阶非线性极 化率, $\mu_{g} \beta$ 值一般均在 $10^{-44} \mathrm{esu}$ 以上; 而通过引入辅助给 体, 分子的 $\mu_{g} \beta$ 值也提高到了 $10^{-44} \mathrm{esu}$ 数量级. 可见, 辅 助给体的引入在提升分子超极化率方面是有极大优势 的. 通过在共轭桥更长的分子(如 EFFC 或 EFNC)中引入 辅助给体，有望得到超极化率更高，同时又能有效防止 结晶的生色团分子.

通过对同一分子上不同辅助给体间的比较发现，首 先，相较于丁氧基和羟基，苠氧基能够更好地作用于这 一类有机非线性生色团分子，使其二阶非线性极化率得 到最大限度的提升; 其次, 丁氧基辅助给体相较于苠氧

表 2 溶剂参数和不同溶剂的紫外-可见频谱

Table 2 Solvent parameters and UV-Vis spectral data in different solvents

\begin{tabular}{|c|c|c|c|c|c|c|c|c|c|c|c|c|}
\hline \multirow[b]{2}{*}{ Solvent } & \multicolumn{3}{|c|}{ Parameter } & \multicolumn{3}{|c|}{ EFC-OH } & \multicolumn{3}{|c|}{$\mathrm{EFC}-\mathrm{OBu}$} & \multicolumn{3}{|c|}{ EFC-OBe } \\
\hline & $\begin{array}{c}\text { Dielectric } \\
\text { const } \varepsilon\end{array}$ & $\begin{array}{l}\text { Refraction } \\
\text { Index } n\end{array}$ & $\begin{array}{l}\text { Density } d / \\
\left(\mathrm{g} \bullet \mathrm{cm}^{-3}\right)\end{array}$ & $\lambda_{\max } / \mathrm{nm}$ & $\begin{array}{c}10^{-4} \varepsilon_{\max } / \\
\left(\mathrm{L} \cdot \mathrm{mol}^{-1}\right. \\
\left.\mathrm{cm}^{-1}\right)\end{array}$ & $\begin{array}{c}10^{-5} \mathrm{C} / \\
\left(\mathrm{mol} \cdot \mathrm{L}^{-1}\right)\end{array}$ & $\lambda_{\max } / \mathrm{nm}$ & $\begin{array}{c}10^{-4} \varepsilon_{\max } / \\
\left(\mathrm{L} \cdot \mathrm{mol}^{-1}\right. \\
\left.\mathrm{cm}^{-1}\right)\end{array}$ & $\begin{array}{c}10^{-5} \mathrm{Cl} \\
\left(\mathrm{mol} \cdot \mathrm{L}^{-1}\right)\end{array}$ & $\lambda_{\max } / \mathrm{nm}$ & $\begin{array}{c}10^{-4} \varepsilon_{\max } / \\
\left(\mathrm{L} \cdot \mathrm{mol}^{-1}\right. \\
\left.\mathrm{cm}^{-1}\right)\end{array}$ & $\begin{array}{c}10^{-5} \mathrm{C} / \\
\left(\mathrm{mol} \cdot \mathrm{L}^{-1}\right)\end{array}$ \\
\hline Ethyl acetate & 6.03 & 1.37239 & 0.9006 & 577 & 1.9075 & 9.15 & 577 & 2.3252 & 8.36 & 574 & 2.8285 & 6.89 \\
\hline Acetone & 20.5 & 1.3588 & 0.7899 & 584 & 2.9228 & 6.62 & 588 & 5.2318 & 3.72 & 591 & 3.2326 & 6.03 \\
\hline DMSO & 48.9 & 1.477 & 1.0958 & 602 & 3.3558 & 5.77 & 607 & 6.5397 & 2.97 & 607 & 5.657 & 3.44 \\
\hline
\end{tabular}


基显示出的性能略有差距, 但这一类生色团分子在溶剂 中的溶解性十分良好, 在制备掺杂型极化聚合物薄膜 时, 或许可以通过加大掺杂质量比而获得更大的宏观电 光系数; 最后, 虽然羟基辅助给体所表现出来的 $\mu_{\mathrm{g}} \beta$ 值 不大, 但由于羟基存在反应活性, 因此也可以考虑将这 一类分子通过 Williamson 反应直接连接到聚合物侧链 上, 制备成侧链型极化聚合物薄膜, 从而可能表现出更 佳的非线性光学性能.

\section{2 结论}

设计并合成了三种含有不同辅助给体的有机非线 性光学生色团分子 EFC-OH, EFC-OBu 和 EFC-OBe. 用 IR 谱、 ${ }^{1} \mathrm{H}$ NMR 谱以及元素分析对生色团分子进行了表 征, 确认其结构正确. 经 TGA 测试结果表明, 三种分子 都具有良好的热稳定性, 加入辅助给体后三分子的热分 解温度均在 $268{ }^{\circ} \mathrm{C}$ 以上, 其中, EFC-OBe 的 $T_{\mathrm{d}}$ 值最高, 为 $278.6{ }^{\circ} \mathrm{C}$. 辅助给体的加入对分子的热稳定性影响不 大. 溶致变色法测试结果表明, 加入辅助给体后, 生色 团分子的非线性光学性能均有较大幅度提高. 其中, $\mathrm{EFC}-\mathrm{OBe}$ 的 $\mu_{g} \beta$ 值最高, 为 $47149 \times 10^{-48} \mathrm{esu}$, 是无辅助 给体的 DCDHF-2-V 分子的 7 倍以上. 综上, 辅助给体的 加入对热稳定性的影响较小, 但能明显能提高了分子的 非线性光学性能. 相比于羟基和丁氧基, 含芐氧基辅助 给体的生色团分子具有更好的热性能和光性能. 这一类 新型的生色团分子具有极好的研究价值与应用前景.

\section{3 实验部分}

\section{1 仪器与试剂}

日本岛津 FTIR-8400 红外光谱仪; Brucker avance300 型核磁共振仪; Vario EL元素分析仪; 北京福凯仪器 公司 X-5 精密显微熔点仪; 热重分析仪(TGA)为美国 TA 公司 Q50 型, 氮气气氛, 流速为 $40 \mathrm{~mL} / \mathrm{min}$, 升温速率 为 $20{ }^{\circ} \mathrm{C} / \mathrm{min}$; 日本岛津公司生产的 UV-2450 型紫外可 见分光光谱仪测定, 工作频率为 $1064 \mathrm{~nm}$.

$\mathrm{TCF}$ 按文献[7]合成, m.p. $200 \sim 203{ }^{\circ} \mathrm{C}$ (文献值 ${ }^{[7]}$ : $200 \sim 203{ }^{\circ} \mathrm{C}$ ); 其它试剂均为市售分析纯.

\section{2 实验方法}

\subsubsection{4- $N, N$-二乙胺基-2-羟基-苯甲醛( $\mathrm{EFO}-\mathrm{OH})$ 的} 合成

在冰浴中, 将三氯氧磷 $20.00 \mathrm{~g}(0.130 \mathrm{~mol})$ 滴入到 $27.78 \mathrm{~g}(0.380 \mathrm{~mol}) \mathrm{DMF}$ 中, 将体系在 $25{ }^{\circ} \mathrm{C}$ 下保温 $1 \mathrm{~h}$, 继续在冰浴中加入 3 -差基- $N, N$-二乙基苯胺 $11.11 \mathrm{~g}$ $(0.067 \mathrm{~mol})$, 连续在 $25,45,65$ 和 $100{ }^{\circ} \mathrm{C}$ 下分别反应 1 , $0.5,0.5,0.8 \mathrm{~h}$ 后, 冷却至室温, 加入 $400 \mathrm{~mL}$ 水, 搅拌 $2 \mathrm{~h}$ 后, 用 $30 \%$ 氢氧化钠水溶液调节 $\mathrm{pH}$ 值到 $4 \sim 5$, 搅拌 0.6 $\mathrm{h}$ 后过滤，用水洗涤滤饼至滤液中性，对所得产物用乙 醇重结晶, 干燥后, 得到无色粉末 $8.02 \mathrm{~g}$, 产率 $62.96 \%$. IR (KBr) v: 2975, 1631, 1560, 1521, 1446, 1417, 1338, 1303, 1240, 1188, 1128, 1080, $1012 \mathrm{~cm}^{-1}$.

3.2.2 2-二氧亚甲基-3-氧基-4-(4-二乙胺基-2-羟基苯乙烯基)-5,5-二甲基-2,5-二氢呋喃(EFC-OH)的合成

取 $0.13 \mathrm{~g}(0.673 \mathrm{mmol}) \mathrm{EFO}-\mathrm{OH}$ 与 $0.10 \mathrm{~g}(0.502$ $\mathrm{mmol}$ ) TCF 于 $20 \mathrm{~mL}$ 乙醇中搅拌, 加热至回流, 回流开 始时滴入 3 滴哌啶作为催化剂, 体系刚开始为浅绿色, 反应 $3 \mathrm{~h}$ 后变为蓝紫色. 反应产物静置 $24 \mathrm{~h}$ 后抽滤, 冷 乙醇洗涤滤饼, 得到蓝紫色固体, 干燥称量得产物 0.14 g, 产率 75.55\%. m.p. $247 \sim 248{ }^{\circ} \mathrm{C} ;{ }^{1} \mathrm{H}$ NMR $\left(\mathrm{CDCl}_{3}\right.$, $300 \mathrm{MHz}) \delta: 1.25$ (t, $J=17.1 \mathrm{~Hz}, 6 \mathrm{H}), 1.55(\mathrm{~s}, 6 \mathrm{H}), 3.45$ $(\mathrm{d}, J=21 \mathrm{~Hz}, 4 \mathrm{H}), 5.35$ (s, 1H), $6.04(\mathrm{~d}, J=2.7 \mathrm{~Hz}, 1 \mathrm{H})$, 6.37 (q, $J=11.1 \mathrm{~Hz}, 1 \mathrm{H}), 6.76 \sim 6.91(\mathrm{~m}, 1 \mathrm{H}), 7.47 \sim 7.61$ $(\mathrm{m}, 1 \mathrm{H}), 8.10(\mathrm{~d}, J=15.6 \mathrm{~Hz}, 1 \mathrm{H}) ;{ }^{13} \mathrm{C} \mathrm{NMR}\left(\mathrm{CDCl}_{3}, 75\right.$ MHz) $\delta$ : 13.6, 16.6, 26.1, 45.0, 45.8, 95.0, 99.9, 101.5, $106.6,107.3,114.4,116.3,129.1,131.2,135.1,151.1$, 159.3, 194.9; IR (KBr) v: 3271, 2983, 2221, 1620, 1529, 1487, 1407, 1342, 1317, 1257, 1186, 1103, $1072 \mathrm{~cm}^{-1}$. Anal. calcd for $\mathrm{C}_{22} \mathrm{H}_{22} \mathrm{~N}_{4} \mathrm{O}_{2}$ : C 70.88, H 5.63, N 14.83; found C 70.57, H 5.92, N 14.96.

\section{$3.2 .34-\mathrm{N}, \mathrm{N}$-二乙胺基-2-丁氧基-苯甲醛(EFO-OBu) 的合成}

取 $0.50 \mathrm{~g}(2.59 \mathrm{mmol})$ EFO-OH 溶于 $10 \mathrm{~mL}$ 丙酮 ${ }^{[28]}$ 中. 另取 $1.07 \mathrm{~g}(7.80 \mathrm{mmol})$ 的碳酸钾加入 $30 \mathrm{~mL}$ 丙酮中 配置成悬浊液, 将两物混合, 在室温下搅拌 $2 \mathrm{~h}$. 然后加 入 $1.07 \mathrm{~g}(7.8 \mathrm{~mol}) \mathrm{C}_{4} \mathrm{H}_{9} \mathrm{Br}$, 回流 $24 \mathrm{~h}$. 蒸去丙酮. 加入 $50 \mathrm{~mL}$ 水, 用二氯甲烷萃取得到棕红色液体, 以体积比 为 $1: 1$ 的二氯甲烷和石油醚为洗脱剂, 柱色谱提纯得 到棕红色液体 $0.45 \mathrm{~g}$, 产率 69.75\%. IR (KBr) v: 2970, 2758, 2358, 1731, 1650, 1519, 1456, 1404, 1338, 1213, $1112,1076,1010 \mathrm{~cm}^{-1}$.

3.2.4 2-二氧亚甲基-3-氭基-4-(4-二乙胺基-2-丁氧 基-苯乙烯基)-5,5-二甲基-2,5-二氢呋喃(EFC-OBu)的 合成

取 $0.45 \mathrm{~g}(1.80 \mathrm{mmol})$ EFO-OBu 和 $0.36 \mathrm{~g}(1.80$ $\mathrm{mmol}) \mathrm{TCF}$ 于 $20 \mathrm{~mL}$ 乙醇中搅拌, 加热至回流, 回流开 始时滴入 5 滴哌啶作为催化剂, 反应 $3 \mathrm{~h}$. 反应产物静置 $24 \mathrm{~h}$ 后抽滤. 得到紫色片状固体, 所得产物用二氯甲烷 为洗脱剂, 柱色谱提纯, 得到紫色固体 $0.48 \mathrm{~g}$, 产率 $61.78 \%$. m.p. $253 \sim 254{ }^{\circ} \mathrm{C} ;{ }^{1} \mathrm{H}$ NMR $\left(\mathrm{CDCl}_{3}, 300 \mathrm{MHz}\right)$ $\delta: 1.02(\mathrm{t}, J=14.7 \mathrm{~Hz}, 3 \mathrm{H}), 1.27(\mathrm{t}, J=14.1 \mathrm{~Hz}, 6 \mathrm{H})$, $1.52 \sim 1.61(\mathrm{~m}, 2 \mathrm{H}), 1.72(\mathrm{~s}, 6 \mathrm{H}), 1.836 \sim 1.927(\mathrm{~m}, 2 \mathrm{H})$, $3.49(\mathrm{~d}, J=21 \mathrm{~Hz}, 4 \mathrm{H}), 4.06(\mathrm{t}, J=12.6 \mathrm{~Hz}, 2 \mathrm{H}), 6.05$ (s, 
1H), $6.36(\mathrm{~d}, J=9.3 \mathrm{~Hz}, 1 \mathrm{H}), 6.84$ (d, $J=15.9 \mathrm{~Hz}, 1 \mathrm{H})$, 7.27 (s, 1H), $7.50(\mathrm{~d}, J=9.3 \mathrm{~Hz}, 1 \mathrm{H}), 8.02(\mathrm{~d}, J=15.9 \mathrm{~Hz}$, $1 \mathrm{H}) ;{ }^{13} \mathrm{C}$ NMR $\left(\mathrm{CDCl}_{3}, 75 \mathrm{MHz}\right) \delta: 14.4,16.1,21.1,27.1$, 33.0, 45.7, 46.9, 70.4, 95.3, 99.5, 102.0, 106.0, 107.9, $115.1,117.8,129.0,132.2,136.0,149.7,157.8,164.3$, 196.2; IR (KBr) v: 2958, 2358, 2223, 1521, 1502, 1427, 1390, 1334, 1313, 1251, 1188, 1157, 1108, 1072, 1016 $\mathrm{cm}^{-1}$. Anal. calcd for $\mathrm{C}_{26} \mathrm{H}_{30} \mathrm{~N}_{4} \mathrm{O}_{2}: \mathrm{C} 72.79, \mathrm{H} 7.15, \mathrm{~N}$ 12.68, found C 72.53, H 7.02, N 13.01.

3.2.5 4- $\mathrm{N}, \mathrm{N}$-二乙胺基-2-芐氧基-苯甲醛(EFO-OBe) 的合成

取 $0.50 \mathrm{~g}(2.60 \mathrm{mmol}) \mathrm{EFO}-\mathrm{OH}$ 和 $1.07 \mathrm{~g}(7.80 \mathrm{mmol})$ 碳酸钾溶于 $10 \mathrm{~mL}$ DMF 中. 在室温下搅拌 $1 \mathrm{~h}$. 然后滴 入 $1.33 \mathrm{~g}(7.80 \mathrm{mmol})$ 溴化芐, 在 $100{ }^{\circ} \mathrm{C}$ 下搅拌 $12 \mathrm{~h}$. 然 后将反应体系倒入冰水中. 静止 $2 \mathrm{~h}$ 后抽滤, 得到不需 再提纯的浅黄色固体 $0.65 \mathrm{~g}$, 产率 $88.65 \%$. IR (KBr) $v$ : 2966, 2837, 1645, 1593, 1523, 1454, 1392, 1274, 1240, $1207,1153,1116,1074,1000,914,808,700 \mathrm{~cm}^{-1}$.

3.2.6 2-二氧亚甲基-3-氭基-4-(4-二乙胺基-2-芐氧 基-苯乙烯基)-5,5-二甲基-2,5-二氢呋喃(EFC-OBe)的 合成

EFC-OBe 的合成方法与 EFC-OH 的合成方法一致, 反应完成后抽滤, 用冷乙醇洗涤滤饼并干燥得到浅绿色 粉末 0.67 g, 产率 $81.74 \%$. m.p. $255 \sim 256{ }^{\circ} \mathrm{C} ;{ }^{1} \mathrm{H}$ NMR $\left(\mathrm{CDCl}_{3}, 300 \mathrm{MHz}\right) \delta: 1.24(\mathrm{t}, J=11.4 \mathrm{~Hz}, 6 \mathrm{H}), 1.56(\mathrm{~s}$, $6 \mathrm{H}), 3.48(\mathrm{~d}, J=6 \mathrm{~Hz}, 4 \mathrm{H}), 5.18(\mathrm{~s}, 2 \mathrm{H}), 6.15(\mathrm{~s}, 1 \mathrm{H}), 6.38$ (d, $J=8.7 \mathrm{~Hz}, 1 \mathrm{H}), 6.76(\mathrm{~d}, J=15.9 \mathrm{~Hz}, 1 \mathrm{H}), 7.26 \sim 7.52$ (m, 6H), 8.08 (d, $J=15.9 \mathrm{~Hz}, 1 \mathrm{H})$; IR (KBr) v: 2979, 2221, 1618, 1525, 1506, 1488, 1425, 1396, 1330, 1249, 1190, $1124,1074 \mathrm{~cm}^{-1}$. Anal. calcd for $\mathrm{C}_{29} \mathrm{H}_{28} \mathrm{~N}_{4} \mathrm{O}_{2}: \mathrm{C} 75.22, \mathrm{H}$ 6.34, N 11.75; found C 74.98, H 6.07, N 12.06.

\section{References}

[1] Feng, J.-K. Acta Chim. Sinica 2005, 63, 1245 (in Chinese). (封继康, 化学学报, 2005, 63, 1245.)

[2] Qi, X.-Y.; Hu, Q.-Y.; Wang, S.-M.; Yang, F.; Yu, P. Acta Chim. Sinica 2012, 70, 363 (in Chinese). (祁小云, 胡泉源, 王世敏, 杨飞, 喻平, 化学学报, 2012, 70, 363.)

[3] Liu, Y.-T.; Wang, X.; Liu, X.-Y.; Ji, Y.-Q. Acta Chim. Sinica 2012, 70, 1131 (in Chinese)

(刘英涛, 王金金, 刘翔宇, 冀永强, 化学学报, 2012, 70, 1131.)

[4] Tang, X. M.S. Thesis, University of Electronic Science and Technology of China, Chengdu, 2010 (in Chinese).

(唐翔, 硕士论文, 电子科技大学, 成都, 2010.)

[5] Li, Q.-Q.; Qin, J.-G.; Li, Z. Chin. J. Org. Chem. 2011, 31, 1337 (in
Chinese).

(李倩倩, 秦金贵, 李振 有机化学, 2011, 31, 1337.)

[6] Tang, X.; Tang, X.-Z.; You, Y.-C.; Ren, L.-K.; Wang, Y.; Yan, L.-J. Acta Chim. Sinica 2012, 70, 1565 (in Chinese). (唐翔, 唐先忠, 游英才, 任立辑, 王洋, 严立京, 化学学报, 2012, 70, 1565.)

[7] Robinson, B. H.; Dalton, L. R.; Harper, A. W.; Ren, A.; Wang, F.; Zhang, C.; Todorova, G.; Lee, M.; Aniszfeld, R.; Garner, S.; Chen, A.; Steier, W. H.; Houbrecht, S.; Persoons, A.; Ledoux, I.; Zyss, J.; Jen, A. K. Y. Chem. Phys. 1999, 245, 35.

[8] Andreu, R.; Cerdán, M. A.; Franco, S.; Garín, J.; Marco, A. B.; Orduna, J.; Palomas, D.; Villacampa, B.; Alicante, R.; Allain, M. Org. Lett. 2008, 10, 4963.

[9] Guieu, V.; Payrastre, C.; Madaule, Y.; Garcia-Alonso, S.; Lacroix, P. G.; Nakatani, K. Chem. Mater. 2006, 18, 3674.

[10] Marder, S. R. Chem. Commun. 2006, 131

[11] Luo, S.-S.; Qiu, Y.-Q.; Liu, X.-D.; Liu, C.-G.; Shu, M.-Z. Acta Phys.-Chim. Sinica 2009, 9, 1867 (in Chinese).

(罗姗姗，仇永清，刘晓东，刘春光，苏忠民，物理化学学报 2009, 9, 1867.)

[12] Liu, J.-S.; Hou, W.-J.; Feng, S.-W.; Qiu, L; Liu, X.-H.; Zhen, Z. J. Phys. Org. Chem. 2011, 24, 439.

[13] Wang, L.; Liu, J.-L.; Bo, S.-H.; Zhen, Z.; Liu, X.-H. Mater. Lett. 2012, $80,84$.

[14] Li, Q.-Q.; Lu, C.-G.; Fu, E.-Q. J. Phys. Chem. B 2008, 112, 4545.

[15] Piao, X.-Q.; Zhang, X.-M.; Inoue, S.; Yokoyama, S.; Aoki, I.; Miki, H.; Otomo, A.; Tazawa, H. Org. Electron. 2011, 12, 1093.

[16] Piao, X.-Q.; Zhang, X.-M.; Mori, Y.; Koishi, M.; Nakaya, A.; Inoue, S.; Aoki, I.; Otomo, A.; Yokoyama, S. J. Polym. Sci., Part A: Polym. Chem. 2011, 49, 47.

[17] Wu, J.-Y.; Liu, J.-L.; Zhou, T.-T.; Bo, S.-H.; Qiu, L.; Zhen, Z.; Liu, X.-H. RSC Adv. 2012, $2,1416$.

[18] Han, L.-K.; Jiang, Y.-D.; Li, W. Mater. Lett. 2008, 62, 1495.

[19] He, M.-Q.; Thomas M. L.; John A. S. Chem. Mater. 2002, 14, 4669.

[20] He, M.-Q.; Thomas M. L.; John A. S. Chem. Mater. 2002, 14, 4662.

[21] Baroja, N. M.; Garin, J.; Orduna, J.; Andreu, R. J. Org. Chem. 2012, 77, 4634.

[22] Tang, X.; Tang, X.-Z.; You, Y.-C. Acta Chim. Sinica 2012, 70, 1565.

[23] Wang, Y.; Tang, X.-Z.; Tang, X. Chem. J. Chin. Univ. 2011, 32, 2327 (in Chinese)

(王洋, 唐先忠, 唐翔, 高等学校化学学报, 2011, 32, 2327.)

[24] Zhan, C.-L.; Wang. D.-Y. Acta Chim. Sinica 2001, 59, 213 (in Chinese). (詹传郎, 王夺元, 化学学报, 2001, 59, 213.)

[25] Pang, M.-L.; Zhang, P.; Dong, Y.-W.; Wang, Y.-M.; Wang, J.-T.; Meng, J.-B. Acta Chim. Sinica 2011, 69, 11 (in Chinese). (庞美丽, 张鹏, 董轶望, 王永梅, 王积涛, 孟继本, 化学学报, 2011, 69, 11.)

[26] Sun, Z.-F.; You, X.-Z.; Li, D.-G. Acta Chim. Sinica 1994, 52, 734 (in Chinese) (孙振范, 游效曾, 李大光, 化学学报, 1994, 52, 734.)

[27] Murugan, N.-A.; Kongste, J. Proc. Natl. Acad. Sci. U. S. A. 2010, 107, 16453.

[28] Paduraru, P. M.; Popoff, R. T.; Nair, R. J. Comb. Chem. 2008, 10, 123. 